\title{
Morale et moralisme à l'école primaire
}

Repères dans une histoire cyclique

\section{Gérard Molina}

\section{CpenEdition}

\section{Journals}

Édition électronique

URL : http://journals.openedition.org/ries/4161

DOI : $10.4000 /$ ries. 4161

ISSN : 2261-4265

Éditeur

Centre international d'études pédagogiques

Édition imprimée

Date de publication : 5 mars 1995

Pagination : 67-85

ISSN : $1254-4590$

Référence électronique

Gérard Molina, "Morale et moralisme à l'école primaire ", Revue internationale d'éducation de Sèvres [En ligne], 05 | 1995, mis en ligne le 16 mars 2015, consulté le 01 mai 2019. URL : http:// journals.openedition.org/ries/4161; DOI : 10.4000/ries.4161

Ce document a été généré automatiquement le 1 mai 2019.

(c) Tous droits réservés 


\title{
Morale et moralisme à l'école primaire
}

\author{
Repères dans une histoire cyclique
}

\author{
Gérard Molina
}

1 Le thème de l'éducation morale couvre deux problèmes corrélatifs mais distincts en droit ${ }^{1}$ . Le problème de la morale de l'école, c'est-à-dire des finalités de l'éducation. Toute société doit se demander quel homme elle veut former et ne peut éluder cette question en la réduisant à une étude instrumentale des techniques et des méthodes pédagogiques. Aristote avertissait : «Il ne faut pas passer sous silence quelle est la nature de l'éducation et de quelle façon elle doit être dispensée (...) si la jeunesse doit cultiver les connaissances utiles à la vie, ou celles qui tendent à la vertu, ou enfin les connaissances sortant de l'ordinaire ${ }^{2}{ }$. Certes, débattre des fins de l'école peut amener la conclusion qu'elles ne s'ordonnent pas principalement à une éthique mais à l'adaptation sociale, à la diffusion de savoirs ou à l'efficacité économique; on reste néanmoins dans un discours anthropologique sur les dispositions et les valeurs qu'une génération estime préférable de développer dans la génération suivante.

2 Le problème de la morale à l'école, c'est-à-dire de savoir si les principes qui régissent les choix pratiques peuvent faire l'objet d'un enseignement séparé dans l'institution scolaire. Paradoxalement, l'école de la République n'a jamais cessé d'être traversée par l'interrogation formulée au début du Ménon par Platon : la vertu peut-elle s'enseigner et s'apprendre, s'acquiert-elle par l'exercice, advient-elle aux hommes par nature ou d'une autre façon? Situons les principales réponses.

3 Pour Ferry et ses continuateurs, comme on va le voir, la morale devait s'enseigner au travers de leçons spécifiques, progressives et ajustées à chaque âge, à côté de l'éducation physique et de l'éducation intellectuelle. Sachant que les autres matières permettent aussi le questionnement éthique, car l'accès aux textes littéraires ou la connaissance historique amènent à s'interroger sur des valeurs, universelles ou particulières à des cultures.

4 Aux yeux des praticiens de "l'école active", au contraire, la morale s'apprend par l'exercice de la vie en commun, l'expérience des contraintes et des bienfaits qu'elle suscite au sein d'un travail coopératif. S'acquérant ainsi par l'épreuve, elle diffère en 
fonction du type de structure collective mise en place et du style pédagogique du maitre, selon qu'on favorise l'autorité magistrale ou l'autonomie de l'élève, l'échange, l'entraide ou la compétition. La classe vise à devenir une communauté rationnelle, construisant la reconnaissance de l'autre dans le partage des tâches.

Ces deux modèles peuvent s'accorder sur certaines vertus à faire acquérir (effort, dignité, honnêteté, franchise, etc.) et s'opposer sur d'autres (l'esprit critique plutôt que la soumission, la solidarité plutôt que la concurrence, etc.).

6 Pour Durkheim, chaque société produit des normes de fonctionnement intégratif s'imposant de l'extérieur à l'enfant comme au maître, qui y sont prédisposés par leurs besoins de régularité, de conformisme et d'altruisme, fruits de leur attachement aux groupes sociaux. Microsociété libérée des enjeux affectifs familiaux, la classe contribue à l'explication rationnelle des règles sociales et à leur intériorisation par les manifestations de la discipline (régularité, habitudes, autorité déléguée par la loi et justifiée par la compétence, punitions et récompenses ostentatoires), ainsi qu'à leur acceptation comme nécessaires et normales par la volonté autonome. La morale est donc relative aux sociétés qui moralisent les individus dès leur naissance, par le langage, les cadres familiaux, les institutions éducatives.

7 Piaget, le critique le plus argumenté du substantialisme holistique durkheimien, a montré que le passage de l'hétéronomie à l'autonomie, d'une morale de la contrainte (expiatoire, rétributive, conformiste) à une morale distributive, réciproque et intentionnelle, pouvait apparaître au cours de jeux et d'activités autonomes ${ }^{3}$. Diverses recherches anglosaxonnes ont raffiné ou corrigé ce modèle de développement progressif des jugements moraux chez l'enfant, en dégageant une échelle précise et rigoureuse de la moralité (décrit en stades successifs, irréversibles et universels), empruntant à divers systèmes philosophiques, grâce à la formulation de dilemmes concrets, anecdotes ou cas: notamment le travail remarquable de Lawrence Kohlberg et de ses continuateurs plus ou moins critiques ${ }^{4}$. Mais, paradoxalement, que le social apparaisse comme une totalité s'imposant de l'extérieur ou comme une dynamique de relations interpersonnelles, l'être humain accède à la morale par une dialectique quasi naturelle entre son être-au-monde et les dispositifs d'acculturation. Le rôle de l'école consiste alors soit à créer des situations favorisant et accélérant la maturation (Piaget), soit à épouser rationnellement le principe d'acceptation des valeurs dominantes (Durkheim); l'autorité du maître découlant du respect qu'il a de sa fonction (Durkheim) ou de son aptitude à collaborer avec les élèves dans la situation ludique (Piaget).

8 Aujourd'hui, ces différentes options circulent dans l'enseignement primaire et parfois, comme c'est souvent le cas pour d'autres démarches éducatives, coexistent dans la même pédagogie.

9 L'éducation morale ne peut donc se satisfaire, comme on le lit ici ou là, d'un banal rappel après une interruption malencontreuse ou d'une restauration à la faveur d'inquiétudes fantasmatiques sur « l'amoralisme de la jeunesse » concomitant à « la baisse du niveau ». On ne saurait faire l'économie de sa déjà longue histoire ni des réflexions qu'elle a produite. Notamment le fait que la question des conditions de possibilité d'une éducation morale à l'école primaire (distincte d'une réflexion sur les fins de l'éthique ou d'une analyse des systèmes de valeurs) décèle aussi une série de paradoxes et d'apories.

10 Comment arbitrer quand l'éducation morale paraît inévitable (puisque le fonctionnement de la classe pose sans cesse des problèmes de justice, de choix entre plusieurs manières 
d'agir, de jugement, d'autorité...) et nécessaire (car que vaudrait une transmission de savoirs et de savoir-faire sans les fins qui sous-tendent leur usage), tout en étant ressentie comme irréalisable (parce que le maître doit proposer, agir, trancher le plus souvent sans expliciter ses critères ni discuter ses valeurs et qu'on attend de lui d'abord une compétence élevée dans les procédures d'apprentissages instrumentaux) ? Comment le même enseignant peut-il articuler le discours positif sur les contenus de l'acte moral et l'effet éthique implicite provoqué par ses interventions (peut-il d'ailleurs éviter toujours la manipulation, le double langage, l'argument d'autorité, etc.) ?

11 N'est-il pas démesuré d'exiger de l'enseignant qu'il édicte des règles de conduite valables pour tous alors que le débat redouble dans la société sur les fondements de la morale, elle-même discutée comme illusion de la conscience, discours idéologique intéressé ou conviction ineffable? Comment échapper à l'alternative entre endoctrinement collectif et relativisme des valeurs ou entre conditionnement caché et pluralisme des codes sinon par une prudente abstention, version appauvrie de la morale (indéfiniment) provisoire de Descartes (sauf que ce dernier admettait des règles pratiques consensuelles alors que les maitres renverraient plutôt aux familles, à la subjectivité ou aux modèles médiatisés) ?

Doit-on inscrire à l'horizon de l'éducation morale l'obtention de réactions conventionnelles à des situations données en fonction des circonstances ou bien de la modification de la personnalité de l'élève guidé vers l'autonomie du jugement fondé sur des principes abstraits universels? On sait en outre que l'amoralisme coexiste parfois avec la réussite scolaire mais est-on sûr que la maîtrise des discours moraux et des axiologies ne peut amener leur utilisation cynique au profit d'un immoralisme aristocratique, leur exploitation hypocrite pour des buts manipulatoires et égoïstes?

Sur un plan factuel, peut-on demander aux maîtres d'éduquer le sens civique d'enfants quotidiennement heurtés par l'étalage des turpitudes auxquelles se livrent des élites en charge du devenir collectif? De quelle autorité la parole du maître sur le bien et sur le mal est-elle alors investie? De la raison pratique, bien sûr, mais que vaut-elle si elle n'oblige pas les détenteurs de la raison pure? La demande pressante de moralisation adressée à l'école (illustrée par le succès public de la série télévisée L'instit) semble la contrepartie de l'érosion du contrat moral par lequel toute société démocratique garantit aux citoyens la réciprocité des devoirs. Lorsqu'une nation exhibe à l'intérieur l'impraticabilité de ses propres lois par les gouvernants et, à l'extérieur, le mariage tératologique de la raison d'État et de l'humanitaire déréalisant, lorsqu'elle érige en finalités suprêmes la réussite financière et la gloire médiatique sanctionnées dans la compétition, que valent les vertus stoïciennes sinon comme vernis culturel, supplément d'âme ou caution de l'ordre établi ?

Ces questions ne nous paraissent ni dépassées ni dilatoires et mériteraient de longs développements. D'autant que le rénovateur le plus éminent d'une morale formaliste, déontologique et universaliste reconnaît qu'enseigner le fondement de la norme éthique ou instruire du jugement sur l'acte juste n'oblige pas à adopter le point de vue moral car "le problème de la faiblesse de la volonté n'est pas résolu par la cognition morale " (Habermas, 1992, p. 169). Admirateur de l'impératif kantien et donc adversaire du néoaristotélisme (affirmant le primat de la valeur sur le juste et l'irréductibilité des modèles de rationalité) et de l'hégélianisme (procureur du formalisme abstrait), il admet pourtant que la force d'agir selon l'intellection morale relève de « la structure de la personnalité et de la forme de vie » que l'école ne peut prétendre maîtriser. 
15 Mais nous voulons ici user d'un éclairage particulier. En effet, le statut d'un enseignement moral réside aussi dans l'explication des tentatives historiques et théoriques auxquelles il doit d'exister comme problème philosophique et pédagogique. D'abord parce que l'institution scolaire française fonctionne largement "à l'amnésie» sur sa propre histoire. Bien que se présentant sans désemparer comme le conservatoire des savoirs et le lieu d'exaltation d'un devoir de mémoire, elle est volontiers oublieuse de ses textes et de ses pratiques, même à courte distance ${ }^{5}$. Ensuite, comme nous le montrerons in fine, parce que certaines prises de position récentes provenant des sciences de l'éducation peuvent ressusciter de vieilles lunes, faute d'un recul historique. Enfin, pour discuter l'idée naïve mais courante que l'éducation morale aurait disparu de l'école par suite d'une démission du corps enseignant. Naturellement, ce regard réflexif sur le passé s'adresse aux éducateurs plutôt qu'aux jeunes éduqués mais tout choix opéré par l'enseignant se déterminerait mieux s'il était informé par le retour sur une tradition qui l'inscrit dans la durée, délivré des astreintes du préjugé commun, de l'urgence médiatique ou de la sommation administrative.

\section{La morale récitée en leçons}

16 L'école républicaine s'affiche anti-platonicienne en matière d'éducation morale. Prenant le contre-pied du Ménon, les textes organisateurs de l'enseignement laïc ont voulu enseigner la vertu (sans toujours s'interroger sur ce qu'elle est) dès l'école primaire publique, lieu principal de sa transmission ${ }^{6}$. Constat ironique si on se rappelle que Socrate s'opposait à la moralité conventionnelle de son temps, jusqu'à en mourir (événement qui livre au passage une définition de la notion de valeur si galvaudée aujourd'hui : une idée de l'homme qui vaut d'être défendue même au prix de la vie ${ }^{7}$ ).

17 L'idée que l'éducation morale puisse faire l'objet d'un enseignement séparé n'a rien d'évident pour les administrateurs des réformes scolaires au XIXe siècle. Si la loi de mars 1850 assure bien que «l'enseignement primaire comprend l'instruction morale et religieuse, la lecture, l'écriture... ", le ministre Fortoul souligne dans ses Instructions du 15 novembre 1854 que les lettres et l'histoire montreront aux élèves le mal et le vice, méprisables et haïssables, et toutes les vertus dignes d'estime et d'amour, ajoutant: «mais il importe d'éviter tout ce qui pourrait en ce genre avoir la moindre apparence d'affectation: l'enseignement moral doit sortir naturellement de la leçon littéraire ». Rédigeant, dans un autre contexte politique, le projet de loi du 15 décembre 1871, Adolphe Thiers et Jules Simon approuvent en précisant : « Quant à la morale, qui doit être l'objet suprême de nos efforts, nous ne chargerons pas nos instituteurs de l'enseigner dans des cours réguliers; nos enfants ne seraient pas en état de les comprendre, et bien peu d'instituteurs seraient préparés à les bien faire; il n'y aura pas, dans les écoles, d'enseignement dogmatique de la morale... ${ }^{8}$ ".

Si les champions de l'ordre moral ne veulent pas d'un enseignement distinct, c'est que la morale est omniprésente dans la relation éducative sous la triple forme de l'éducation religieuse (quotidienne), de la soumission au maître (modèle des autres assujettissements à tous les types d'autorité) et de l'exigence d'inculcation dans «l'âme des enfants du sentiment de ce qu'ils doivent à Dieu, à leurs parents, au chef d'État et à leurs semblables ${ }^{9}$ ». L'élève (et le futur adulte travailleur) n'a que des devoirs (" d'obéissance, de probité, de dévouement ») ; l'instituteur est lui aussi défini par le devoir d'être " exemplaire » afin de mieux graver ces devoirs dans les consciences enfantines. Mais à force d'être partout 
présente dans l'enseignement, la morale ne risquait-elle pas d'être nulle part? Ainsi infusée, imprégnait-elle les actes quotidiens ou ne devenait-elle pas évanescente? Quoiqu'il en soit, cette attitude offrait surtout le bénéfice de ne pas hasarder la définition d'une morale distincte des dogmes chrétiens, ni causer la confrontation des options philosophiques.

Les doctrinaires républicains innovèrent en sécularisant les règles de la vie commune et en faisant de l'école primaire le lieu d'une transmission comparable aux (mais dissociée des) autres apprentissages. Ils mirent le problème éthique au centre de la philosophie républicaine (d'où sortirent les contributions de Guyau, Fouillée, Buisson ou Durkheim), renouant avec l'idéal de l'État-nation éducateur. En distinguant morale profane et morale confessionnelle, ils installaient une double révolution - réalisée depuis longtemps dans le champ philosophique - consistant d'une part à montrer que l'homme pouvait être juste et charitable sans la crainte de Dieu ni l'espérance d'un salut, d'autre part à séparer épistémologiquement le domaine «des croyances, qui sont personnelles, libres et variables, et celui des connaissances, qui sont communes et indispensables à tous " (J. Ferry, 17 novembre 1883). De plus, ils articulèrent les devoirs de l'individu et les droits du citoyen comme fondement d'une anthropologie politique. Pourtant, ils n'échappèrent pas à la contradiction en caractérisant la morale comme un impératif catégorique s'imposant de lui-même à la conscience tout en requérant la totalité de l'appareil scolaire pour simplement advenir ${ }^{10}$.

20 Le philosophe Paul Janet, dans son Plan d'instruction morale dans les écoles primaires (21 juin 1882), expose la première alternative en rejetant «cet enseignement diffus et presque inconscient de la morale » au profit «d'un petit cours particulier donné en dehors des autres matières d'enseignement sous des formes aussi simples et aussi modestes que possible, mais enfin un cours où la morale sera enseignée pour elle-même ${ }^{11}$ ». Mais il ajoute que, la morale reposant sur les idées les plus naturelles et conformes à l'âme humaine, "il ne s'agit que de les réveiller, de les provoquer », plutôt que de les faire admettre et partager. Dans sa Lettre aux Instituteurs (17 novembre 1883), Ferry parle aussi d'un enseignement qui n'apporte " rien de nouveau, rien qui ne vous soit familier comme à tous les honnêtes gens ». D'ailleurs les programmes de 1887 sont plus volontaristes en projetant « d'en faire une habitude qui gouverne la vie (...), d'incliner la volonté libre vers le bien ». L'imposition de cette nouvelle culture dicte à l'instituteur "de former chez l'enfant le sens moral, de l'aiguiser, de le redresser parfois, de l'affermir toujours ». Une nouvelle matière d'enseignement est née et l'élève deviendra autre qu'il aurait été sans l'école publique. La morale de l'école ne s'épuise pas dans celle du "père de famille ", comme le prétend Ferry (d'ailleurs attaqué sur ce point par Durkheim).

21 Cette innovation marque l'école française jusqu'à aujourd'hui car on ignore trop que l'éducation morale ne disparut jamais des programmes du primaire. Les Instructions de 1887, 1923, 1938, 1969 et 1977-80 réaffirment son importance même si sa portée, son contenu et sa place dans le cursus subirent des modifications qu'il est impossible de retracer dans le cadre de cet article ${ }^{12}$. Le ministre Chevènement n'a donc pas rétabli l'éducation morale et civique en 1985, il en a inversé la logique. Alors qu'au xix ${ }^{e}$ siècle, le civisme est pour l'essentiel une extension de la vertu privée, dans les Programmes de 1985, l'éducation civique est en elle-même "éminemment morale " et "développe l'honnêteté, le courage, le refus des racismes, l'amour de la République ${ }^{13}$ ». La III République avait moins besoin de citoyens acteurs de la société civile que de travailleurs respectueux des normes sociales; le pouvoir moderne suppose qu'en prenant conscience 
des mécanismes juridiques de l'État démocratique, le citoyen intériorisera les conduites qui en permettent la pérennité.

Parallèlement, les derniers programmes mettent au premier plan les règles de fonctionnement de la vie collective au sein du groupe-classe ${ }^{14}$

Le curriculum du premier degré défini par la loi du 28 mars 1882 affirmait: "L'enseignement primaire comprend l'instruction morale et civique ; la lecture... », etc. Une priorité essentielle illustrée par le fait que Gabriel Compayré - universitaire et député, spécialiste d'histoire de l'éducation - publia le premier manuel des classes élémentaires en 1881, un an avant l'adoption des grandes lois républicaines ${ }^{15}$. L'ambition était double : désarmer la critique cléricale qui voyait dans l'enseignement laïc d'État la fabrication de demi-savants sans foi ni loi, mais aussi remplacer partiellement la direction de conscience appuyée naguère sur le catéchisme et l'hagiographie. En substituant «l'instruction civique » à "l'instruction religieuse ", les républicains déplacèrent en fait l'accent sur «l'instruction morale » qui, d'introuvable, devenait le pivot du système.

Pour Compayré, l'instituteur ne doit pas seulement transmettre des savoirs fondamentaux, il a "une mission sociale », il a «charge d'âmes» (p. ix). L'opposition sempiternellement ressassée entre instruire et éduquer n'existe absolument pas (ni rhétoriquement, ni conceptuellement) chez les doctrinaires de l'école républicaine : « Que l'instituteur ne craigne donc pas d'étendre son action, de contribuer à l'éducation morale, comme à l'instruction positive et technique de ses élèves (...) il doit être de plus en plus le maitre de la jeunesse, l'instituteur de la conscience et du cœur de ses enfants » (p. x). Au reste, l'amour du maître pour ses élèves (et réciproquement) fait intégralement partie de la relation pédagogique.

Aussi bien, les Instructions de 1887 distinguent soigneusement le caractère de l'éducation morale des autres parties du programme qui reposent sur la précision et la liaison logique des vérités : « la force de l'éducation morale dépend (...) de l'intensité du sentiment, de la vivacité des impressions et de la chaleur communicative de la conviction ». Ayant pour objectif d'inscrire dans la conscience enfantine une disposition au vouloir, elle a affaire avec l'intime, mobilise la source des émotions et l'élan du cœur, non d'abord pour convaincre mais pour capter ${ }^{16}$.

On trouve chez Compayré d'autres affirmations qui contredisent l'image de l'école républicaine que des polémiques récentes ont $\mathrm{cru}$ bon de donner ${ }^{17}$. Inversement, beaucoup de raisonnements par les tenants actuels de la rénovation pédagogique datent au moins de 1880. Ainsi, l'école de Jules Ferry ne se présente pas en institution conservatrice ou dissociée, elle justifie au contraire par la modernité les innovations proposées. Justification utilitariste : «Il est certainement plus intéressant et plus utile de savoir comment s'est formé le village ou la ville que nous habitons que de connaître l'histoire de la création du monde. Mieux vaut encore se rendre compte des attributions du maire de sa commune que savoir qu'il y a eu des juges dans les tribus d'Israël et des consuls à Rome» (p. 41).

Refus également de la coupure entre la société et l'école qui sont dans une relation de microcosme à macrocosme car "la vie scolaire est en petit l'image de la vie sociale » (caractérisée par un but commun, le respect des chefs et l'assistance mutuelle); les mêmes fonctions, les mêmes normes et les mêmes valeurs s'y déploient nécessairement (p. 42-43). S'adressant à un nouveau public dont ils devaient faire leur base électorale, les 
idéologues républicains présentaient leur réforme scolaire comme une adaptation au monde moderne.

Mais le discours éthique des débuts de la III ${ }^{e}$ République recèle de fortes tensions internes. Y compris chez un même auteur, tel Ferry qui écrit à l'instituteur: "Votre rôle en matière d'éducation morale est très limité ", tout en exigeant qu'il produise dans l'enfant «tous les signes d'une incessante amélioration morale ». Ou encore Compayré : d'un côté, le régime républicain réclame des citoyens éclairés sur les principes qui gouvernent la souveraineté qu'ils devront exercer (mais Compayré cite plus volontiers Pestalozzi que Condorcet et compare l'instruction du citoyen-électeur à l'exercice du soldat $\left.{ }^{18}\right)$; de l'autre, la morale privée suffit à faire le bon citoyen car « le meilleur principe des vertus civiques sera toujours la pratique des vertus individuelles » (p. Ix et 133). L'école forme le citoyen conscient... qui l'est déjà en étant honnête homme. De la justice interindividuelle à la justice civique, aucune solution de continuité ${ }^{19}$.

D'une part, Compayré affirme que les intelligences enfantines sont plus capables qu'on ne l'imagine « de prendre goût à des études où sont engagés ses intérêts futurs d'homme et de citoyen » (p. IX). Mais d'autre part, l'enfant (jusqu'à sa majorité) est contraint à une soumission absolue, aveugle et volontaire : «l'enfant doit obéir à ses parents en toutes choses (...) obéir même aux ordres dont il ne comprend pas la raison (...) non pas seulement en apparence, mais de toute son âme, sans murmure et sans résistance " (p. 21). Exercer son jugement, fonder en raison ses prises de parti, former son esprit critique devraient surgir d'un abandon de la compréhension! D'autant que « l'instituteur est le remplaçant des parents", les enfants de la classe sont "comme une famille d'adoption» (p.35) et chaque enfant doit aimer ses parents, même s'ils paraissent indifférents ou ont "mauvais caractère " (p.15). Rendant la fréquentation scolaire obligatoire, la République garantit et relaye l'autorité parentale (certes définie par le Code civil).

Cette éducation aux règles sociales dessine en creux une société vue comme un système de rapports interindividuels commandés par un double statut immuable, d'égal à égal, d'inférieur à supérieur. Ainsi, l'enfant est supérieur aux animaux, égal de ses frères et de ses camarades, inférieur à ses maîtres et parents. Tel parent est supérieur à ses enfants mais inférieur à ses chefs naturels que sont le patron, le maire, le juge. L'école inculque le respect, défini comme « l'aveu que l'on fait de son infériorité et se manifeste par les actes, par les paroles qui conviennent vis-à-vis d'un supérieur » (p. 22). Le patriotisme s'oppose au cosmopolitisme car la solidarité entre Français est supérieure à la fraternité des peuples et on doit « préférer la patrie » à l'humanité (p. 59).

31 C'est que l'histoire s'arrêtait là. En effet, contrairement à ce qu'on lit encore, aux yeux de Compayré et de ses amis, l'égalité fonde les autres valeurs (liberté, fraternité, solidarité) car elle « est le principe de la société civile telle que l'a faite la Révolution ». En brisant les « inégalités de convention» et en abolissant les privilèges, «la Révolution n'a laissé subsister que les inégalités inévitables qui dépendent de la nature ", c'est-à-dire les différences individuelles dans la capacité de travail, l'intelligence ou le talent. L'égalité n'est donc pas une promesse ou une espérance, le dernier des objectifs de la démocratie, mais bien une réalité quotidiennement maintenue par l'acte inaugural.

En faisant de la morale un enseignement parmi les autres : « il y a un apprentissage de la vertu comme de toutes choses » (p. 126), les pédagogues républicains prenaient le risque, malgré qu'ils en aient, de le rabaisser au niveau des autres. Apprentissage verbal et 
formel puisque, sonder les cœurs ou fouiller les consciences étant impossibles, son évaluation se limite à faire réciter les règles et les exemples. Aussi, le maître laïc doit dans ce domaine lorgner vers son prédécesseur et concurrent tonsuré en recommandant comme moyens de perfectionnement moral de faire chaque jour son "examen de conscience », d'admirer les «grands hommes » et de méditer « les belles maximes » (id.). Plus explicite, Janet demandait aux instituteurs d'imiter «les jeunes prêtres» qui réussissent « à pratiquer la prédication, à donner des instructions morales et religieuses ${ }^{20}$ ». Pragmatique, Jules Ferry dans sa Lettre tirait le maître vers « l'îlotier » vérifiant si une leçon sur la loi empêchait les élèves « au sortir de la classe de commettre une fraude, un acte, fût-il léger, de contrebande ou de braconnage ", ce qui signifierait l'échec du cours ${ }^{21}$.

Par-dessus tout, la place de Dieu dans l'architectonique de la morale laïque divisait les fondateurs de l'école républicaine. Pour Janet, «le couronnement naturel de cette instruction morale devrait être la connaissance de Dieu. On apprendra aux enfants que la vie a un but sacré, que les hommes ne sont pas le produit du hasard, qu'une pensée sage veille sur l'univers et qu'un œil vigilant pénètre dans toutes les consciences». Cette religion naturelle providentialiste prépare logiquement le terrain des religions positives « car l'État ne s'engage pas à soutenir que la piété purement intérieure est suffisante, et il laisse aux différents cultes à montrer qu'elle ne l'est $\operatorname{pas}^{22}$ ». Janet attendait que la morale profane encourage les âmes à se tourner vers les dogmes révélés. Inversement, les Programmes de 1887 rappellent à l'instituteur que l'immense majorité des enfants lui arrive " ayant déjà reçu ou recevant un enseignement religieux qui familiarise avec l'idée d'un Dieu auteur de l'univers et père des hommes ». On lui recommande donc de mûrir cette connaissance et de la faire passer dans la pratique quotidienne.

Pour Compayré, Dieu est une puissance plus lointaine dont les avatars terrestres (parents, maître, chefs) occupent le devant de la scène. Il figure comme garantie ultime mais indispensable. En revanche, aux yeux de Ferdinand Buisson, la morale scolaire ignore Dieu car elle vise à faire aussi bien que la religion mais sans l'appui d'aucune transcendance, en se fondant sur la puissance de «l'intuition morale » qui est, comme l'acquisition d'une langue maternelle, un produit de l'évolution de l'espèce humaine (à la fois héritable et perfectionnable). Etre juste, pour Buisson, c'est « être dans l'état normal (...) de l'action vitale ». Dans son article du Dictionnaire, Buisson opère un double mouvement: 1) en tant que scientiste, il accepte l'idée d'une «morale scientifique » que la sociologie énoncera un jour prochain ; 2) comme idéologue du progrès biologique, il croit à l'existence d'une morale qui s'impose naturellement au petit humain, sans raisonnement, sans recours à la peur, la punition ou l'intérêt, sans puissance extrinsèque, "phénomène d'atavisme bien qu'il ait la spontanéité apparente d'un mouvement instinctif ${ }^{23} »$.

L'examen des centaines d'ouvrages consacrés à la morale scolaire révélerait bien d'autres désaccords, tensions ou contradictions, suivant que les auteurs s'adressaient aux adversaires cléricaux, à l'opinion publique ou aux formateurs, selon les systèmes philosophiques auxquels ils empruntaient leurs argumentaires, selon les objectifs pédagogiques poursuivis et surtout les contextes historiques successifs.

\section{L'ère du soupçon}

Rendons grâce cependant à l'optimisme énergique des pédagogues républicains. Il permit que, durant une longue période, sur toute l'étendue d'un territoire, à tous les niveaux de 
l'instruction populaire, plusieurs générations d'élèves reçurent un enseignement détaillé, imaginatif et ajusté des grands énoncés consensuels dégagés par la réflexion morale. Pour la première fois, on fut témoin de l'expérimentation grandeur nature d'une initiation méthodique aux vertus laïques (obéissance, effort, travail, excellence, respect). Mais on dut constater aussi sa banqueroute au fur et à mesure qu'instituteurs et anciens élèves prirent conscience très progressivement, à la faveur de quelques événements politiques majeurs (guerre, crise et effondrement de l'État) et en fonction d'autres facteurs irrésumables ici, que la morale civique était bafouée par ses représentants, que les magistères moraux ignoraient l'équité et que l'intelligence pouvait cohabiter avec l'ignominie.

Dans la première moitié $d u \mathrm{Xx}^{\mathrm{e}}$ siècle, l'éducation morale si précise, sérieuse et impérative ressemble déjà tellement à un vestige fossilisé qu'un auteur comme Alain, pourtant inquiet de voir l'utile prendre le pas sur le sacré, n'y consacre que quelques pages dans ses Propos sur l'éducation, proposant de la résumer en une seule maxime: "Ce que tu conseilles à ton voisin, fais-le » (LII). C'est à notre avis dans la littérature qu'on trouve les meilleurs témoignages d'une perte de confiance dans le théorème qui voulait que la connaissance de la vertu et la compréhension rationnelle du bien développent une traduction pratique chez des êtres instruits et éduqués ensemble.

En 1928, un fils de « hussard noir » formé à l'École normale obtient un triomphe théâtral avec une pièce qui raille l'éducation morale dont les préceptes servent à dissimuler le fonctionnement social réel. Topaze met en scène un enseignant d'une institution privée qui prépare ses élèves à une « composition de morale » conforme aux programmes : « La question que vous aurez à traiter demain, et qui décidera de votre rang, ne sera pas une question particulièrement limitée comme le serait une question sur la patrie, le civisme, les devoirs envers les parents ou les animaux. Non. Ce sera plutôt, si j'ose dire, une question fondamentale sur les notions de bien et de mal, ou sur le vice et la vertu. Pour vous préparer à cette épreuve, nous allons nous pencher sur les mœurs des peuples civilisés, et nous allons voir ensemble quelles sont les nécessités vitales qui nous forcent à obéir à la loi morale, même si notre esprit n'était pas naturellement porté à la respecter ${ }^{24}$ ». Dans une salle de classe tapissée d'aphorismes édifiants, Topaze explique que la société récompense l'honnête homme selon son mérite tandis que le riche malhonnête, privé de l'approbation de sa conscience, vit entouré de faux amis, vrais courtisans. Mais après dix années de cette défense d'une justice immanente, il est remercié pour avoir refusé d'améliorer les notes d'un cancre fortuné. Ce renvoi plonge Topaze dans les tripatouillages de la politique et des affaires où il finit par troquer sa moralité naïve contre une réussite financière éclatante qui lui apporte la considération des notables, l'amour des femmes et même les palmes académiques. À son ancien collègue, il explique cyniquement que « l'argent peut tout, permet tout, donne tout » et qu'il montrerait à ses élèves que «le mépris des proverbes c'est le commencement de la fortune » (acte IV, scène 4.).

Pagnol ne fait pas que transposer dans l'univers scolaire les intrigues du théâtre réaliste contestataire illustré par Henri Becque ou Octave Mirbeau, il dresse le bilan de l'instruction civique après la boucherie de 1914-1918. Parmi les illusions balayées par la cruauté d'une guerre que rien ne vient justifier mais dans laquelle anciens élèves et professeurs ont payé le prix fort, figure en bonne place la croyance qu'une solide éducation rendrait les hommes plus fraternels et la société plus solidaire. À la mise à nu de l'hypocrisie des valeurs sociales, Pagnol ajoute le dévoilement de la duplicité qui 
cimente le système : les puissants enseignent aux dominés comment ils doivent vivre en vivant eux-mêmes selon des normes exactement inverses. Mal payé, ignorant la vie réelle, glosant sur les plaisirs spirituels, le maître d'études incarne la vertu mais se fait le complice involontaire d'un mensonge avilissant pour lui et ses élèves (sauf ceux, issus d'un milieu bourgeois, qui balancent pour savoir si leur répétiteur est un imbécile ou s'il se moque d'eux). Pagnol n'avait pas besoin de caricaturer les visées pédagogiques de son personnage pour déclencher le rire d'un public scolarisé entre 1890 et 1920. En effet, l'éthique républicaine revendiquait bien une finalité hédoniste et Compayré promettait dans son manuel la joie spinoziste ou l'eudémonisme aristotélicien : « la morale, en vous enseignant comment vous devez vous conduire en toute circonstance, n'a pas seulement pour but de faire de vous des hommes honnêtes, elle a aussi pour objet de vous rendre heureux (...) elle est aussi la science du bonheur» (p. 105-106). Tout comme Voltaire se moquait dans Candide d'un énoncé leibnizien prétendant circonscrire l'expérience du monde, Pagnol riait du catéchisme moral kantien infantilisée ${ }^{25}$.

Rien de plus axiologique que ce message : les intérêts ne s'opposent pas aux valeurs car la valeur de l'existence réside dans la satisfaction des intérêts (indissociablement spirituels et matériels), dans le conatus qui la fait persévérer dans son être égoïste, la joie par l'accomplissement de soi, et parallèlement l'acceptation de la cruauté de l'existence. La morale aristocratique des forts commence toujours par diaboliser la morale du ressentiment des faibles: vous niez les lois réelles de la vie parce que vous n'avez ni l'énergie ni l'intelligence pour les affronter.

41 C'est qu'une nouvelle interrogation taraudait l'enseignant : en n'apprenant pas aux élèves les ressorts vrais qui commandent la réalisation des valeurs du moi, ne manquait-il pas à sa mission de préparation au monde réel ? Les maîtres qui abandonnèrent peu à peu l'éducation formelle de la morale ne le firent pas d'abord au nom d'une introuvable «liberté » de l'enfant ou d'une préséance des familles, mais par refus de l'hypocrisie sociale et de leur propre instrumentalisation dans la diffusion d'une morale dogmatique dont les prescripteurs s'estimaient exemptés. D'ailleurs, loin de devenir cyniques, beaucoup d'entre eux prirent tellement au sérieux l'espérance éthique qu'ils crurent possible de l'établir dans une société plus juste et se firent militants.

Par surcroît, Pagnol exhibait le lien entre les maximes inculquées à l'élève et les obligations pesant sur le maître. Car l'éducation morale se doublait d'un contrôle de soi et d'une ascèse professionnelle qui avaient laissé une empreinte profonde.

\section{Déontologie du maître d'école}

En effet, jusqu'à la création des IUFM (1991), le programme de formation des Écoles normales comportait une rubrique "Déontologie du métier d'instituteurs". Ordinairement assuré par les professeurs de philosophie, cet « enseignement » ne laissait pas de surprendre à plusieurs titres. Réflexion supposée sur les devoirs du maître, la déontologie ne se confond pas avec l'éducation morale ni avec la morale comme question philosophique (deux autres points du programme). D'ailleurs, à la différence de ceux-ci, le thème est ignoré des bibliographies ou des index se rapportant à l'éducation. Inscrite dans la préparation d'une profession peu sujette aux faits divers, la déontologie était en revanche absente d'autres formations à des métiers (ceux de policier, journaliste, ingénieur commercial ou homme politique) beaucoup plus exposés à des manquements et risques variés. En France, les écoles de police n'introduisirent la déontologie qu'en 1986, 
les écoles de commerce ont mollement importé "l'éthique appliquée des affaires", développée sous forme d'une casuistique aux États-Unis, les écoles de journalisme ont pris récemment conscience du problème, enfin l'ENA et l'Institut des sciences politiques estiment toujours leurs étudiants immunisés. De plus, aucun code de déontologie ne régente le métier d'instituteur alors que les activités soumises à un code officiel (médicales, juridiques ou d'expertises) le réduisent souvent à la portion congrue dans leur cursus $^{26}$.

Forgé au début du XIX $\mathrm{X}^{\mathrm{e}}$ siècle par Jeremy Bentham, le terme déontologie désigna rapidement l'étude des principes qui gouvernent l'action des individus mais en tant qu'ils agissent en fonction de leur appartenance à un groupe professionnel déterminé27. Cette invention est liée à l'essor de métiers appelés à jouer un rôle central dans la régulation des rapports marchands. D'une certaine façon, l'individualisme libéral opérait une synthèse empirique entre hétéronomie et autonomie de la morale, puisque les membres d'un groupement professionnel se donnaient librement des règles de conduite qui s'imposaient à eux et aux impétrants tout en en comprenant les raisons déduites des conditions d'exercice de leur activité. D'un autre point de vue, il laissait supposer que les conduites justes du citoyen et celles de certains membres spécialisés différaient, en raison d'exigences particulières ou d'une image de leur fonction. L'existence d'une éthique professionnelle crée le dualisme d'un droit commun et d'un droit particulier dont la concordance demeure précaire. Même codifiées, les déontologies admettent l'adaptation à l'évolution du droit et de l'opinion, parfois difficilement (les médecins avaient un article contre l'avortement). En ce sens, elles sont plus proches des lois civiles que des lois morales tout en se référant volontiers à des principes universels.

Si la déontologie restait incluse dans la formation de maîtres, c'est que l'autorité administrative avait depuis toujours lesté l'instituteur d'un fatras de devoirs qui dénotaient la suspicion. On peut en prendre la mesure à la lecture du fameux mais peu étudié Code Soleil ${ }^{28}$. Sa première partie concernait les "directives de la morale professionnelle dégagée des textes promulgués » et les « principes que nul ne doit ignorer et qu'il n'est pas possible d'enfreindre ». On rappelle à l'instituteur qu'il est le « représentant de la nation » (p. 46) pour mieux obtenir l'intériorisation d'une discipline personnelle constante, érigée en surmoi. Une formule résume tout ce que l'autorité attend de lui : "l'instituteur a donc l'obligation de se montrer particulièrement sévère pour lui-même (...) il lui faudra éviter jusqu'à l'apparence d'un abandon» (p. 30). Deux obsessions vertèbrent son rôle: d'une part "tous ses faits et gestes sont soumis au contrôle public» (id.); d'autre part, non seulement "pas une minute l'attention des élèves ne doit s'évader » dans la classe (p. 12), mais le maître doit « tout faire pour que les élèves ne soient pas entièrement livrés à eux-mêmes en dehors des heures de classe, non plus que les anciens élèves durant la période qui sépare l'école du régiment » (p. 51). Sans cesse placé sous le regard de tous, il incarne la neutralité de l'État, doit exhiber une conduite irréprochable, éviter à la fois l'indifférence et l'arrogance, surtout s'interdire quantité de choses (depuis les « parties de plaisir trop fréquentes » et les « fréquentations douteuses » jusqu'aux opérations commerciales, y compris la vente de manuels scolaires, en passant par l'acceptation de cadeaux qui « aliènent une part de sa dignité et de son indépendance »). Il va de soi que "l'institutrice, surtout, aura à se surveiller» (p. 30). De plus, la scolarisation du territoire a fixé un maitre en chaque point d'un maillage serré; or « c'est un coin de France qu'on lui confie. Il va en être l'éducateur, le moralisateur, le philosophe » (p. 8); les maitres sont donc poussés à assurer un très grand nombre 
d'œuvres péri- et postscolaires qui prolongent la présence de l'État et empêchent le désœuvrement et la liberté des enfants (p. 50-52).

En lisant la liste des maximes que l'enfant devait respecter et celle des devoirs prescrits aux instituteurs jusqu'à une date récente, le Code Soleil n'abandonne ces recommandations qu'à la fin des années 1960, on se prend à paraphraser doublement Beaumarchais : « Aux vertus qu'on exigeait d'un élève, connaît-on beaucoup de maitres qui fussent dignes d'être élèves? Aux vertus qu'on exigeait d'un maître d'école, connait-on beaucoup de ministres de l'éducation qui fussent dignes d'être instituteurs ${ }^{29}$ ? ». Mais si ces obligations du maître n'avaient cessé d'enfler au cours du XXe siècle, c'est que la moralisation de l'élève était plus attendue du style magistral (image de soi, climat de classe, autorité naturelle, incarnation de la justice, etc.) que d'une didactique explicite des valeurs et des devoirs. En mettant en avant la découverte des normes de la vie commune au travers des activités du groupe, on transférait la morale d'un corps de séquences objectives à l'attitude pédagogique et affective de l'enseignant. Mais s'appuyer sur la dynamique propre à la classe pour instaurer des règles de vie ne peut pas faire oublier que le groupe est aussi le lieu des conflits d'intérêt, des luttes de pouvoir, des distorsions de la communication et des projections affectives.

Surtout, Bentham et les républicains étaient réunis par la conviction que les mécanismes sociaux réels (poursuite de l'intérêt individuel ou solidarité croissante) contribuaient naturellement à l'essor d'une moralité publique que l'effort scolaire confortait et anticipait à la fois. Or, la mise en crise de cette conviction conduit aujourd'hui à des réinterprétations surprenantes du rôle de la morale à l'école, comme en témoigne un article-manifeste publié dans Le Monde (2 avril 1993) et signé de cinq éminents universitaires avec le titre éloquent «Oser éduquer ${ }^{30}$ ». Leur argumentation ne laisse pas d'étonner. Puisque la société moderne produit inévitablement «le morcellement identitaire", "le repli sécuritaire», "la fragmentation sociale», "l'exclusion de l'emploi », l'école forme l'ultime rempart face à cette désintégration des valeurs et des solidarités, «en première ligne dans la bataille toujours recommencée pour la cohésion sociale ». En érigeant en axiome une impuissance avouée face à une logique économique mortifère ( des dangers nouveaux qui touchent à la nature même de notre société se profilent à l'horizon du siècle»), ils magnifient l'éducation qui à la fois cimente et subsume les deux autres finalités de l'école, l'instruction (exerçant la raison) et la formation professionnelle. La vision fataliste de l'évolution sociale hypostasie la mission fondamentale de l'école : enseigner « les valeurs, les règles, les interdits, les devoirs - en un mot la morale » ou « le sens moral », bref « éveiller à la conscience morale ${ }^{31}$ » car de nombreuses familles ont cessé de transmettre «les interdits fondamentaux du meurtre, de l'inceste ou du vol » (spécialement dans les banlieues).

Dans une nation parcourue de forces centrifuges, les maitres auraient pour fonction principale de freiner un délitement rendu inévitable par l'évolution socio-économique. Paradoxe: quoiqu'obsédés par l'actualité, les auteurs ne s'aperçoivent pas qu'ils reproduisent le discours de la pédagogie des jésuites (la vie sociale entretenant la corruption des mœurs, l'éducation doit fournir à l'enfant le modèle d'une société idéale fondée sur la justice en l'arrachant aux influences délétères), sauf que ces derniers, plus cohérents, concluaient à la fermeture scolaire alors que nos pédagogues modernes prônent, comme on sait, l'ouverture de l'école. Ouvrir l'école sur la société qu'ils décrivent devient un cauchemar injustifiable. 
49 Attaquant sévèrement les défenseurs de l'instruction suffisante (traités de «naïfs ou d'apprentis sorciers »), Meirieu et ses collègues les accusent de préparer une société sans éthique, au titre qu'on rencontre partout des gens instruits mais mal éduqués (à tous les sens du mot) et des nouveaux barbares dépourvus des contrôles de soi primaires. La morale est ainsi instrumentalisée pour diaboliser l'adversaire: qui ne l'installe pas au premier rang de son projet éducatif, contribue à détruire la société, rien de moins! Pourtant, ils écrivent que c'est le fonctionnement social qui détruit la société civile et non les partisans de l'instruction. Comment «refonder sans cesse une société solidaire et fraternelle» si les mécanismes internes de cette société reposent sur l'exclusion et la fragmentation, comment faire adhérer à des règles et des valeurs partagées si elles sont bafouées et détruites par « la logique économique »?

Pour Meirieu et ses collègues, il existe une morale distincte, claire et univoque mais plus personne ne prend la peine de l'exposer aux enfants. Non seulement ils oblitèrent le caractère problématique des conceptions éthiques, mais ils s'offrent au pouvoir d'état comme auxiliaires indispensables du consensus et du lien social que ce même pouvoir est incapable d'assurer. Légitimer l'éducation morale par la hantise des conséquences sociales d'un fonctionnement économique qu'on a au préalable naturalisé, réifié et transformé en destin peut séduire une autorité dont on espère avantages et gratifications, mais cela se traduit inéluctablement par une exhortation à la résignation et une prédication pleine de bons sentiments.

51 Au moins, Ferdinand Buisson savait « qu'une morale vaut ce que vaut la civilisation dont elle est le résumé » et qu'existe dans la société française «à la base de tout, même de la morale, ce grand fait de l'inégalité sociale persistante». Il ajoutait que l'égalité et la liberté n'existent qu'en droit car «beaucoup ne sont libres que de mourir de faim ou de végéter dans une situation misérablement précaire. Ils ne sont égaux aux autres qu'un instant tous les quatre ans devant l'urne». C'est pourquoi il refusait que "la morale populaire » se contente de conformer la raison pratique des enfants aux lois et usages du moment, mais ouvre sur une "raison mystique» qui rendrait chacun capable «de protester contre l'égoïsme de la société actuelle au nom d'un rêve que réalisera la société future ${ }^{32} »$.

On peut préférer, avec la tradition épicurienne, penser que la condition d'une vie heureuse repose sur la destruction des espoirs fallacieux, des mensonges et des illusions, sur l'acceptation lucide et totale du réel, mais il faut alors fournir à l'élève les moyens d'une compréhension rationnelle du monde, d'une libération des tyrannies de l'affect et de la pulsion, d'un détachement vis-à-vis de l'utilité immédiate et de l'actualité obsédante. Bref, l'arracher à lui-même et l'aider à se construire comme humain soucieux de vérité et de liberté.

Si bien que le paradoxe ultime de l'éducation morale aujourd'hui, c'est qu'elle concerne sans doute plus les adultes que les enfants. 


\section{NOTES}

1. O. Reboul, Les Valeurs de l'éducation, PUF, 1992. J.C. Forquin, «L'enfant, l'école et la question de l'éducation morale. Approches théoriques et perspectives de recherches ", Revue française de pédagogie, 102, 1993, p. 69-106 (remarquable bibliographie). J.J. Wunenburger, Questions d'éthique, PUF, 1993 (panorama réflexif des problématiques de la philosophie morale).

2. Aristote, Politique, VIII, 1337 a, trad. Tricot, Vrin, 1970, p.553-554. Aristote savait que les opinions différaient sur la vertu et les moyens de l'acquérir ou que les cités grecques proposaient plusieurs types de formation en fonction de leur système politique et des mœurs. Il défendait une éducation publique unifiée et croyait, bien avant Durkheim, qu'un citoyen appartient d'abord à l'État.

3. E. Durkheim, L'éducation morale, 1925 ; J. Piaget, Le jugement moral chez l'enfant, 1932.

4. Les œuvres de Kohlberg n'ont pas été traduites mais on peut en trouver des synthèses et des commentaires dans les textes de Forquin et Reboul (note 1) ainsi que dans Bril et Lehalle, Le développement psychologique est-il universel ?, PUF, 1988, ou Jürgen Habermas, De l'éthique de la discussion, trad. M. Hunyadi, Éd. du Cerf, 1992 (notamment p. 50-91) et Morale et communication, trad. Éd. du Cerf, 1986.

5. Nous pourrions citer cent exemples de ce perpétuel effacement du passé digne du 1984 d'Orwell (la destruction des traces en moins). Notons seulement que la "réintroduction » de l'éducation morale fut proposée par le ministre Beullac et donna lieu à un rapport officiel du ministère de l'éducation, Enseignement et valeurs morales. Combats contre tes ombres, 1981, La Documentation française.

6. Rappelons que les Républicains instituèrent deux réseaux scolaires étanches et qu'il faudrait donc pouvoir comparer avec l'enseignement moral des petites classes des lycées et des premières années de l'enseignement secondaire (existait-il ?).

7. Les sophistes prétendaient former à la vertu, moins en apportant une éloquence moraliste qu'en jouant sur l'ambiguïté d'un mot (arete) dont le contenu se transformait, passant de l'excellence militaire à l'exemplarité civique.

8. On retrouvera ces textes fondateurs, et beaucoup d'autres, dans le livre de Claude Bernard, Comment nos ministres font l'histoire, Presses Universitaires du Mirail, 1990, cit. p. 39, 70-71. Thiers et Simon ajoutaient: "l'enseignement régulier de la morale, qui serait déplacé et peut-être dangereux dans les écoles, tiendra désormais le premier rang dans les écoles normales ». Pour les enfants, la morale devait apparaître comme une nécessité naturelle, une fonction organique de l'existence aussi indiscutable que la respiration.

9. Règlements pour les écoles publiques, recueil des actes administratifs de la préfecture de la Seine, le $1{ }^{\mathrm{e} r}$ octobre 1855. op. cit. p. 40. Le cas de l'école maternelle relève d'une étude à part car on y «moralise » surtout par l'hygiène et l'habitude.

10. C'est pourquoi beaucoup prônaient en sus une « éducation de la volonté » pour laquelle Jules Payot proposa, dans divers ouvrages, justificatifs et techniques

11. Le rapport de Janet, présenté devant la section permanente du Conseil supérieur de l'Instruction publique, est particulièrement reproduit dans l'entrée «Morale et civique (instruction) » du Dictionnaire de Pédagogie et d'instruction de F. Buisson (1er éd. 1887, nous citons l'édition de 1911). Ce long article donne une idée très précise et détaillée du type d'enseignement moral (procédés, méthodes, leçons, exemples, etc.) que les maîtres devraient délivrer depuis la maternelle jusqu'au cours supérieur, ainsi que le texte des Programmes et instructions de 1887. 
12. II existe plusieurs recueils des textes administrant l'école primaire. Ici, Michel Salines, Pédagogie et éducation, Mouton, 1972, p. 9-52.

13. Les programmes de 1985 et 1986, toujours en vigueur (1994-95), ont été repris intégralement dans la «nouvelle politique» des cycles à l'école primaire (1990) qui les développent sous la forme de " compétences à acquérir ». En réalité, ces dernières les transforment sans le dire (par exemple, disparition des "symboles de la République ", de la notion de patrie, de la devise républicaine, etc. au profit de la connaissance de la CEE ou de la SNCF). Comparer les pages 118-120 et 65 de la brochure Les Cycles à l'école primaire, CNDP-Hachette, 1991.

14. Pour une réflexion sur les rapports entre morale civique et morale individuelle, voir les articles rassemblés par la revue Spirale, $\mathrm{n}^{\circ} 7$, janv. 1992, EN-Lille.

15. Gabriel Compayré, Éléments d'éducation civique et morale, 1881. Deux fascicules, l'un pour «le degré élémentaire ", l'autre pour "le degré moyen et supérieur». Comme il s'agit d'un enseignement à la fois progressif et concentrique, nous citons le second qui approfondit les mêmes conceptions. Cet ouvrage, sans cesse réédité durant quarante ans, fut précédé de plusieurs interventions programmatiques, telle la conférence de Paul Bert, L'instruction dans une démocratie (Le Havre, 21 mars 1880). Les fondateurs de l'école républicaine parlaient indistinctement d'instruction, d'éducation ou d'enseignement moral et civique.

16. Un autre passage de Compayré est encore plus explicite : "L'instruction est un ensemble de connaissances, l'éducation un ensemble de bons sentiments, de bonnes habitudes morales. L'instruction fait l'homme capable, l'éducation le fait honnête. L'instruction donne la science, l'éducation la sagesse. Ce sont les exemples et les conseils de famille, les enseignements religieux, les leçons de morale qui contribuent le plus à l'éducation.»(p. 33). Voir aussi p.35-37. Complémentarité totale.

17. Ce constat ne signifie naturellement pas que les réflexions sur l'école rassemblées par Hannah Harendt et développées en France, sous des points de vue parfois différents, par J. Muglioni, J.C. Milner, C. Kintzler soient dépourvues de sens et de vérité. Mais il était illusoire et suicidaire de les transposer, comme certains l'on fait avec une intention politique de continuité imaginaire, dans la III ${ }^{e}$ République. Par certains côtés, leurs conceptions sont plus novatrices et dérangeantes que la vulgate pédagogique qui soumet l'acte éducatif à l'opinion commune et aux besoins de la cité.

18. Op.cit. p.185. Les pédagogues d'aujourd'hui amalgament rhétoriquement les attitudes intellectuelles (curiosité, esprit critique, amour de la vérité...) et les dispositions morales (entraide, honnêteté, désintéressement...) comme si l'activité scientifique supposait toujours une éthique de la recherche consubstantielle.

19. Naturellement, il faudrait considérer les pratiques réelles de classe. L'ouvrage de Compayré comporte 102 leçons articulées dans une progression.

20. Paul Janet, op. cit., p. 1353.

21. Ferry proposait sa grille d'évaluation des séquences de morale : «avouer une faute, (...) se refuser à une indélicatesse ou à un passe-droit » ou encore aider un camarade en péril plutôt que de ne songer qu'à soi-même. Petit dilemme à usage des ferristes : doit-on aider le camarade en perdition le jour de la composition?

22. Paul Janet, op.cit. p. 1352. Janet s'oppose aux adversaires d'un enseignement moral en soutenant que ce serait laisser le domaine spirituel aux seuls religieux.

23. Article « Morale » du Dictionnaire de pédagogie et d'instruction, $2^{\mathrm{e}}$ éd., p. 1348-1352

24. Marcel Pagnol, Topaze, acte I, scène 12. Cette pièce connut un triomphe (alors que Les marchands de gloire, satire du patriotisme d'État, rencontra l'échec en 1925) et fut jouée dans toutes les capitales européennes; on en connaît au moins sept adaptations cinématographiques (trois en France, une aux États-Unis, en Chine, en Égypte et en Grande-Bretagne). L'autre œuvre théâtrale importante sur l'éducation est sans doute La leçon d'Eugène Ionesco (1951) dans laquelle le maître est naturellement conduit à tuer son élève. 
25. Le cours « imité du catéchisme moral de Kant » est proposé par Compayré dans son manuel p.112-114. Là encore, il ne craint pas de se contredire en admettant que les circonstances peuvent faire que «le bonheur échappe même au plus vertueux des hommes " qui n'a alors d'autre ressource qu'en « appeler à l'espoir d'une autre vie, compter sur la justice de Dieu ». Le déisme républicain retrouvait les postulats de la raison pratique (Dieu, la liberté et l'immortalité) par un biais singulier.

26. Depuis 1947, les codes déontologiques sont introduits dans le droit positif sous forme de règlements d'administration publique. Celui des avocats exige le «désintéressement, la modération » et l'exactitude aux audiences (décret du 10 avril 1954, art. 16). Le rôle des conseils de l'Ordre mériteraient de longues remarques. La réflexion déontologique est peu développée en France. Voir néanmoins l'article «Déontologie» dans l'Encyclopedia Universalis, la revue Esprit, décembre 1990.

27. II faut donc distinguer la déontologie du déontologistes, conception apparue au début du $\mathrm{xx}^{\mathrm{e}}$ siècle selon laquelle l'action morale est régie par l'obligatoire ou le juste et non le bien ou l'utile. Cf. Paul Ricœur, Lectures 1, Seuil, 1991, p. 182 et John Rawls, Théorie de la Justice, trad. 1987.

28. Le Code Soleil doit son nom à Joseph Soleil, chef de bureau au ministère de l'Instruction publique et chargé de conférences de législation scolaire aux Écoles normales supérieures de Fontenay et Saint-Cloud, qui publia son premier Livre des instituteurs en 1923. La 56 édition parut en 1990 sous la plume d'une équipe issue du SNI-PEGC. Nous citons ici une édition intermédiaire, la $13^{\mathrm{e}}$ de 1939 , mais il faudrait consacrer une étude aux transformations du métier d'instituteur reflétées par les modifications de ce volumineux traité législatif, administratif et pédagogique.

29. Beaumarchais, Le barbier de Séville, acte I, scène II, où Figaro ironise : « on veut que le pauvre soit sans défaut ». Dans son monologue du Mariage de Figaro, il montre que, pour gagner du bien, le savoir-faire vaut mieux que le savoir et que chacun est occupé à piller autour de lui en exigeant qu'il soit honnête.

30. Les auteurs sont A. Bouvier, M. Fort, B. Gelas, Ph. Meirieu et J.-P. Obin qui déclinent leur titres et fonctions en fin d'article. Pour abréger nous retiendrons le nom de Meirieu qui signe là sans doute une synthèse de ses propositions.

31. Le texte-manifeste est saturé par ces qualificatifs d'autant plus remarquables que $\mathrm{Ph}$. Meirieu est aujourd'hui membre du comité de rédaction des Cahiers pédagogiques, revue qui titra en avril 1981 «L'école en danger de morale» ( $\left.n^{\circ} 113\right)$, suite à la proposition du ministre Beullac de réactiver l'éducation morale!

32. Buisson, Dictionnaire..., p. 1350. Par ces affirmations, il détonne au sein des autres pères fondateurs de l'école républicaine ; le manuel de Compayré enseigne « que les pauvres se gardent de tout sentiment d'envie et de rancune contre les riches » (p. 132).

\section{RÉSUMÉS}

Une étude richement informée de l'histoire de l'instruction morale en France depuis la création de l'école laïque, qui défait une image convenue et simpliste des intentions des fondateurs. L'échec final de cette expérience atteste l'impossibilité de stabiliser une formation morale et civique à l'école dont l'idéal soit en contradiction manifeste avec la réalité des pratiques sociales ordinaires. 
INDEX

Index géographique : France, Europe

Mots-clés : école primaire, éducation morale, histoire de l'éducation, valeur, système éducatif, éducation

\section{AUTEUR}

\section{GÉRARD MOLINA}

Professeur de philosophie, Institut universitaire de formation des maîtres, Paris, France 